\title{
A NEWTON-GMRES APPROACH FOR THE ANALYSIS OF THE POST-BUCKLING BEHAVIOR OF THE SOLUTIONS OF THE VON KÁRMÁN EQUATIONS
}

\author{
KOKOU DOSSOU* \\ AND ROGER PIERRE \\ DÉPARTEMENT DE MATHÉMATIQUES ET DE STATISTIQUE, \\ UNIVERSITÉ LAVAL, QUÉBEC, CANADA, G1K 7P4
}

\begin{abstract}
We propose a Newton-GMRES type algorithm to solve the discrete von Kármán problem. We show that this algorithm is efficient both in memory and computation time and robust in the neighborhood of the singular points of the bifurcation diagrams. Placing ourselves in the context of the Schaeffer and Golubitsky theory, we use this algorithm to study the post-buckling behavior of a rectangular plate clamped and compressed along its four sides.
\end{abstract}

Key words. von Kármán equations, finite element, mode interaction, mode jumping

AMS subject classifications. 65N30, 65F10, 35B32, 65N25, 74K20

1. Introduction. Consider a thin flat rectangular plate

$$
\Omega=\{(x, y) \mid 0 \leq x \leq \ell, 0 \leq y \leq 1\}
$$

subjected to a uniform compression applied in the normal direction to a portion of the boundary.

Starting from rest, a continuous increase of the lateral compression will eventually result in large displacements which may be modeled by the von Kármán equations,

$$
\left\{\begin{array}{l}
\Delta^{2} u=[\lambda \theta+\phi, u]+f, \quad \text { in } \Omega, \\
\Delta^{2} \phi=-[u, u], \quad \text { in } \Omega, \\
u=\frac{\partial u}{\partial n}=0, \quad \text { on } \partial \Omega \\
\phi=\frac{\partial \phi}{\partial n}=0, \quad \text { on } \partial \Omega
\end{array}\right.
$$

In (1.1), $u$ denotes the deflection, that is to say the displacement in the normal direction to the median plate, [.,.] denotes the Poisson bracket defined by

$$
[u, v]=\frac{\partial^{2} u}{\partial x^{2}} \frac{\partial^{2} v}{\partial y^{2}}+\frac{\partial^{2} u}{\partial y^{2}} \frac{\partial^{2} v}{\partial x^{2}}-2 \frac{\partial^{2} u}{\partial x \partial y} \frac{\partial^{2} v}{\partial x \partial y},
$$

and $\phi$ is the Airy stress potential. The parameter $\theta$ is a regular function defined on the whole of $\Omega$, the values of which depend only on the portion of the boundary subjected to compression or, equivalently, on the boundary conditions. The bifurcation parameter $\lambda$ is a measure of the intensity of that compression and, from the stability analysis point of view, one is mainly interested in the modifications of the solution set of (1.1), as $\lambda$ is slowly increased.

From the point of view of continuum mechanics, (1.1) is a simplified model. For example, the physical parameters of the plate such as Young Modulus or the Poisson ratio are absent, a simplification which is valid only for polygonal plates. Furthermore, the model does not take into account the possibility of rotations, which would require

\footnotetext{
*TO WHOM CORRESPONDENCE SHOULD BE ADDRESSED
} 
a shell model. Nevertheless, for the analysis of the post-buckling behavior of an isotropic nonlinear thin plate, a comparison between results obtained with this model and a shell model shows little difference in the quantitative results, at least for the first bifurcation values [25]. This model also plays a role in mechanical problems. For example, it appears as an intermediate step in the study of viscoelastic plates [3] and, more recently, it has been playing a major role in the analysis of thin film blistering $[29,20]$, an application field of growing importance.

However, from the mathematical point of view, which we adopt here, its main interest lies in the fact that its solutions, considered as functions of the compression and of the length exhibit all the characteristic behaviors of those of more complicated nonlinear thin structure models: bifurcations, mode jumping, snap-through, etc. The mathematical importance and wealth of this model is illustrated in many publications both of a theoretical and computational nature. In fact, even if analytical approaches can be used on simple geometries [14], any deep exploration of the solutions of the system requires a more sophisticated toolbox, which must include efficient and robust numerical algorithms such as the one described below.

It has long been observed that in (1.1) the variables $u$ and $\phi$ are only weakly coupled, since $\phi$ depends linearly on $[u, u]$ through the biharmonic operator. Indeed, if we denote by $\Delta_{\omega}^{-2}$ the inverse biharmonic operator with boundary conditions associated to $\phi$, we can rewrite (1.1) in the following compact form

$$
\left\{\begin{array}{l}
\Delta^{2} u=\left[\lambda \theta-\Delta_{\omega}^{-2}[u, u], u\right]+f, \quad \text { in } \Omega, \\
u=\frac{\partial u}{\partial n}=0, \quad \text { on } \partial \Omega,
\end{array}\right.
$$

Equation (1.2) is clearly cubic in $u$ and this observation has served as the basis of most of the analytical studies of the existence and multiplicity of the solutions. Surprisingly it does not seem to have been considered as a basis for numerical computation. In the following, we shall show that the application of a Newton-GMRES type method to (1.2) leads to a solver which is both efficient in memory and computation time and robust in the neighborhood of singular points of the bifurcation diagram. This being done, we shall use this algorithm to study the behavior of a rectangular plate clamped and compressed along its four sides. Such a situation is not really physically realistic, however it is mathematically very intriguing, in that the topology of the bifurcation diagrams is much more intricate than in the case of the plate clamped only along its shortest sides.

Here is a brief outlook of the paper. In the next section, we shall study the linearized problem around $u=0$ and define both the finite element discretization and the basic iterative algorithm, making a few comparisons with other approaches that can be found in the literature. The nonlinear variant will be introduced in section 2 , where we shall detail the full algorithm. The problem of numerical detection of bifurcation points will be quickly considered in section 3 , whereas section 4 will be devoted to a complete numerical analysis of the fully clamped plate.

2. The basic algorithm for the linearized problem. In a previous paper [13], we have presented a complete toolbox that can be used to obtain a full picture of the solution set of (1.1). This toolbox is based on a combination of the analytical approach of [14] and various numerical procedures. As is often the case, the weak point was the computation cost. 
A stability analysis of a one parameter nonlinear model, written in a general form as

$$
\mathcal{F}(\mathbf{X}, \lambda)=0
$$

is usually undertaken with some continuation method, the most often used being the Euler-Newton algorithm of Keller [17], in which $\lambda$ is considered as an extra unknown determined by choosing the arc length $s$ as the solution curve parameter. In this approach, one moves from one solution corresponding to a value $s_{0}$ to a neighboring one corresponding to $s_{0}+\delta s$ in two steps:

- prediction of a new solution by following the tangent line (Euler step);

- correction of the solution using some nonlinear iterative solver (Newton).

If, in the first step, one chooses to follow the exact tangent, one has to solve a linear system. However, in many practical situations, a simpler approximation by a secant line proves to be sufficient. In any case, the bulk of the computational time is spent in the second step. To use a Newton corrector with exact resolution of the linearized problem at each iteration, one has then to solve two linear systems. This is a consequence of the continuation approach since the addition of one unknown changes the tangent matrix $d_{X} \mathcal{F}$ into

$$
\left(\begin{array}{cc}
d_{X} \mathcal{F}(X(s), \lambda(s)) & d_{\lambda} \mathcal{F}(X(s), \lambda(s)) \\
X^{\prime}(s) & \lambda^{\prime}(s)
\end{array}\right) .
$$

This precludes standard $L U$ factorization which would result in unwanted fill-in and some block LU factorization must be used. Even in that case, the tangent matrix $d_{X} \mathcal{F}$ will have to be assembled and factorized at each Newton iteration.

In the context of discretized PDE systems, this approach is very costly mainly because of the size of the nonlinear problem. Some alternatives have thus been proposed. A general study of iterative resolution of the bordered system can be found in [6] but the methods proposed there require matrix manipulations that are unrealistic in our context. The use of Krylov-based methods is advocated in [16] and the resulting algorithm is applied to a fluid mechanics problem. For the von Kármán problem per se, a nonlinear conjugate gradient has been studied in [24], which allows for a reduction of the matrix size and which is computationally efficient away from the bifurcation points. Unfortunately this algorithm is not robust since convergence is rather slow in the neighborhood of a singular point. In a very recent paper, Chien et al. [8] have proposed a Block-GMRES method in which the two systems that have to be solved at each Newton iteration are solved simultaneously with a ILU-preconditioned GMRES approach. The method seems to be effective but a lack of documentation of the various control parameters does not allow precise quantitative comparisons. Moreover, since the condition number of the matrix involved depends in a crucial way on the chosen discretization, any direct speed of convergence comparison is bound to be incomplete.

In essence our approach is similar to the above in that we start from the EulerNewton algorithm but try to improve the correction step by replacing the direct solver by an iterative one. Here as in [8], the corrector is still of Newton type but the solver is no longer direct. In fact, in [8], even the philosophy of block factorization is 
preserved, since two systems are solved simultaneously whereas in our approach we consider the bordered system, more in the Reinhart fashion. Nevertheless, as in [24], the system is solved by a procedure which allows for a reduction of the number of effective unknowns by a direct substitution approach in which the computation of the various residuals is performed in several steps.

For the sake of clarity, it will be easier to start by having a look at the linearized version of (1.1), around a solution $u=0$.

Find $u \in H_{0}^{2}(\Omega)$ such

$$
\left\{\begin{array}{l}
\Delta^{2} u-\lambda[\theta, u]=f, \quad \text { in } \Omega, \\
u=\frac{\partial u}{\partial n}=0, \quad \text { on } \partial \Omega .
\end{array}\right.
$$

For a rectangular domain, many different discretization procedures can be applied to (2.2). It is not our goal here to discuss merits and defects of those procedures and we shall limit ourselves to saying that the validity of a mixed finite element approach is well documented. Indeed, variational discretization of the standard biharmonic problem by $C^{0}$ finite elements has been the subject of an extensive literature (see [12] and the references therein). In [13], we have shown that, among these, the Miyoshi formulation in which the auxiliary variable is the full Hessian, is particularly well adapted to a stability analysis since it leads to a straightforward numerical evaluation of the relevant analytical parameters. As to the theoretical aspects, first considered in [19], they have been fully revisited in [22]. Thus, here we shall limit ourselves to a brief sketch.

2.1. The variational formulation. For this, we need to recall a few facts about 2-tensors.

1. The deviator $\Upsilon(H)$ of a 2 -tensor $H$ is defined by

$$
\Upsilon(H)=H-\frac{1}{2} \operatorname{trace}(H) I_{2}
$$

2. The scalar product of two symmetric tensors $H$ and $G$ is given by

$$
H: G=H_{1,1} G_{1,1}+H_{2,2} G_{2,2}+2 G_{1,2} H_{1,2} \text {. }
$$

3. If we define a symmetric indefinite product by

$$
\llbracket H, G \rrbracket=H_{1,1} G_{2,2}+H_{2,2} G_{1,1}-2 G_{1,2} H_{1,2} .
$$

we get

$$
\llbracket H, G \rrbracket=\frac{1}{2} \operatorname{trace}(H) \operatorname{trace}(G)-\Upsilon(H): \Upsilon(G) .
$$

4. When a tensor $H$ is symmetric, so is its deviator. Hence, if we set

$$
\Upsilon(H)=\left(\begin{array}{cc}
\mathcal{S}_{1}(H) & \mathcal{S}_{2}(H) \\
\mathcal{S}_{2}(H) & -\mathcal{S}_{1}(H)
\end{array}\right)
$$

we have, for the scalar product of two deviatoric tensors

$$
\Upsilon(H): \Upsilon(G)=2\left(\mathcal{S}_{1}(H), \mathcal{S}_{1}(G)\right)+2\left(\mathcal{S}_{2}(H), \mathcal{S}_{2}(G)\right)
$$


Now, let two functions $u, v \in H^{2}(\Omega)$ be given, and $H(u)$ and $H(v)$ denote their Hessians. Noting that $\Delta u=\operatorname{trace}(H(u))$, and that

$$
\mathcal{S}_{1}(H(u))=\frac{1}{2}\left(\frac{\partial^{2} u}{\partial x^{2}}-\frac{\partial^{2} u}{\partial y^{2}}\right), \quad \mathcal{S}_{2}(H(u))=\frac{\partial^{2} u}{\partial x \partial y},
$$

it is immediately verified that the Poisson bracket can be rewritten as

$$
[u, v]=\llbracket H(u), H(v) \rrbracket .
$$

Since deviatoric tensors will play a role in our discretization, we define for any subspace $X$ of $L^{2}(\Omega)$ the space

$$
\Sigma_{D}(X)=\left\{\left[\begin{array}{cc}
s_{1} & s_{2} \\
s_{2} & -s_{1}
\end{array}\right] \mid s_{1} \text { and } s_{2} \in X\right\} .
$$

Now setting $w=\Delta u, \mu=\Upsilon(H(u))$ and using (2.4), we may consider the following mixed variational formulation of $(2.2)$

Find $(u, w, \mu) \in H_{0}^{1}(\Omega) \times H^{1}(\Omega) \times \Sigma_{D}\left(H^{1}(\Omega)\right)$ such that

$$
(2.5)\left\{\begin{array}{l}
a_{2}(\mu, \nu)+b_{2}(\nu, u)=0, \quad \forall \nu \in\left(H^{1}(\Omega)\right)^{2} \\
a_{1}(w, \psi)+b_{1}(\psi, u)=0, \quad \forall \psi \in H^{1}(\Omega) \\
b_{1}(w, v)-\lambda\left(\frac{1}{2} \int_{\Omega} \Delta \theta w v d \underline{x}-\int_{\Omega}(\Upsilon(\theta): \mu) v d \underline{x}\right)=\int_{\Omega} f v d \underline{x}, \forall v \in H_{0}^{1}(\Omega) .
\end{array}\right.
$$

where the bilinear forms $a_{1}, a_{2}, b_{1}$ and $b_{2}$ are defined by

$$
\begin{aligned}
a_{1}: L^{2}(\Omega) \times L^{2}(\Omega) & \longrightarrow \mathbb{R} \\
\left(\psi_{1}, \psi_{2}\right) & \longmapsto \int_{\Omega} \psi_{1} \psi_{2} d \underline{x}, \\
a_{2}: \quad \Sigma_{D}\left(L^{2}(\Omega)\right) \times \Sigma_{D}\left(L^{2}(\Omega)\right) & \longrightarrow \mathbb{R} \\
(\mu, \nu) & \longmapsto \int_{\Omega}(\mu: \nu) d \underline{x}, \\
b_{1}: \quad H^{1}(\Omega) \times H^{1}(\Omega) & \longrightarrow \mathbb{R} \\
\left(\psi_{1}, \psi_{2}\right) & \longmapsto \int_{\Omega} \nabla \psi_{1} \nabla \psi_{2} d \underline{x},
\end{aligned}
$$

and

$$
\begin{array}{cl}
b_{2}: \Sigma_{D}\left(H^{1}(\Omega)\right) \times H_{0}^{1}(\Omega) & \longrightarrow \mathbb{R} \\
(\nu, v) & \longmapsto \int_{\Omega} \operatorname{div} \nu \cdot \nabla v d \underline{x} .
\end{array}
$$

In (2.5), the first two equations are expressing the fact that $\mu=\Upsilon(H(u))$ and $w=\Delta u$ whereas the last one is nothing but the PDE itself where $\Delta^{2} u=\Delta(\Delta u)$ and the Poisson bracket has been expressed in terms of the deviator and trace of $H(u)$. Note that the integrals $\int_{\Omega} \Delta \theta w v d \underline{x}$ and $\int_{\Omega}(\Upsilon(\theta): \mu) v d \underline{x}$ are defined if $\theta$ is in $C^{2}(\Omega)$. 
2.2. The finite element approximation and its matrix form. In [24] a first-order finite element discretization of (2.5) has been proposed. However, as was shown in [22] this choice imposes a severe restriction on the mesh, too severe in fact for the discretization to be of any practical value. This is the reason for which we choose a second-order discretization, the construction of which is straightforward. Given a triangulation $\mathcal{T}_{h}$ of $\Omega$, we define the spaces

$$
\begin{aligned}
& \mathcal{X}_{h}=\left\{\psi_{h} \in C^{0}(\bar{\Omega}) ;\left.\psi_{h}\right|_{T} \in P_{2}, \quad \forall T \in \mathcal{T}_{h}\right\}, \\
& \mathcal{V}_{h}=\mathcal{X}_{h} \cap H_{0}^{1}(\Omega)
\end{aligned}
$$

and the discrete problem: find $\left(u_{h}, w_{h}, \mu_{h}\right) \in \mathcal{V}_{h} \times \mathcal{X}_{h} \times \Sigma_{D}\left(\mathcal{X}_{h}\right)$ such that

$$
\left\{\begin{array}{l}
a_{2}\left(\mu_{h}, \nu_{h}\right)+b_{2}\left(\nu_{h}, u_{h}\right)=0, \quad \forall \nu_{h} \in \Sigma_{D}\left(\mathcal{X}_{h}\right) \\
a_{1}\left(w_{h}, \psi_{h}\right)+b_{1}\left(\psi_{h}, u_{h}\right)=0, \quad \forall \psi_{h} \in \mathcal{X}_{h} \\
b_{1}\left(w_{h}, v_{h}\right)-\lambda\left(\frac{1}{2} \int_{\Omega} \Delta \theta w_{h} v_{h} d \underline{x}-\int_{\Omega}\left(\Upsilon(\theta): \mu_{h}\right) v_{h} d \underline{x}\right) \\
=\int_{\Omega} f v_{h} d \underline{x}, \forall v_{h} \in \mathcal{V}_{h} .
\end{array}\right.
$$

Problem (2.6) has an interesting block-matrix form. Indeed, if we let $U, W, S_{1}$ and $S_{2}$ be the nodal value vectors of the corresponding variables, we may rewrite it as

$$
\left[\begin{array}{cccc}
M & 0 & 0 & B_{2}^{1} \\
0 & M & 0 & B_{2}^{2} \\
0 & 0 & M & B \\
\lambda D_{\theta}^{1} & \lambda D_{\theta}^{2} & \lambda C_{\theta}+B^{T} & 0
\end{array}\right]\left[\begin{array}{c}
S_{1} \\
S_{2} \\
W \\
U
\end{array}\right]=\left[\begin{array}{c}
0 \\
0 \\
0 \\
F
\end{array}\right]
$$

If $S_{1}$ and $S_{2}$ are extracted from the first two equations and substituted in the remaining, the system is further reduced to

$$
\left[\begin{array}{cc}
M & B \\
\lambda C_{\theta}+B^{T} & -\lambda\left(D_{\theta}^{1} M^{-1} B_{2}^{1}+D_{\theta}^{2} M^{-1} B_{2}^{2}\right)
\end{array}\right]\left[\begin{array}{c}
W \\
U
\end{array}\right]=\left[\begin{array}{c}
0 \\
F
\end{array}\right] .
$$

To keep the mathematical expressions from becoming lengthy we introduce the following notations:

$$
A_{\lambda}=\left[\begin{array}{cc}
M & B \\
\lambda C_{\theta}+B^{T} & -\lambda\left(D_{\theta}^{1} M^{-1} B_{2}^{1}+D_{\theta}^{2} M^{-1} B_{2}^{2}\right)
\end{array}\right], \quad X=\left[\begin{array}{c}
W \\
U
\end{array}\right], \quad G=\left[\begin{array}{c}
0 \\
F
\end{array}\right] .
$$

Equation (2.8) is thus written in compact form

$$
A_{\lambda} X=G
$$

2.3. The linear solver. $A_{\lambda}$ not being a sparse matrix, a direct method is not a reasonable option. $A_{\lambda}$ being non-symmetric the conjugate gradient method is not readily applicable either. So we choose to use a preconditioned GMRES as our solver, which means that we have to consider preconditioning. Using

$$
P_{\lambda}=\left[\begin{array}{cc}
M & B \\
\lambda C_{\theta}+B^{T} & 0
\end{array}\right]
$$


as preconditioner we get the system

$$
P_{\lambda}^{-1} A_{\lambda} X=P_{\lambda}^{-1} G
$$

In order to avoid the factorization of $P_{\lambda}$ for each value of $\lambda$ it is tempting to replace $P_{\lambda}$ by $P_{0}$. We have done that and could not find any significant difference in the performance of the algorithm even far away on the bifurcating branches. This excellent behavior might be explained in the following heuristic way. Looking at the definition of $P_{0}$, one can reinterpret (2.9) as the discretized version of a simple modification of $(2.2)$ which can be written as

$$
u=\Delta_{u}^{-2}(\lambda[\theta, u]+f)
$$

where $\Delta_{u}^{2}$ is the biharmonic operator with clamped boundary conditions. Thus, the choice of $P_{0}$ corresponds to a preconditioning at the continuous level and it appears that our choice of discretization gives rise to a particularly well conditioned matrix.

2.4. A numerical test. Before moving on to the nonlinear case, we quickly present a validation of the algorithm when applied to $(2.2)$

For this, let us consider a rectangular plate $\Omega=] 0, l[\times] 0,1[$ (with $l=3.9$ ) subjected to a uniform lateral compression on its two ends. In that case $\left(\theta=-\frac{1}{2} y^{2}\right)$ (see $[10])$. The linearized problem is thus written

$$
\left\{\begin{array}{l}
\Delta^{2} u+\lambda \frac{\partial^{2} u}{\partial x^{2}}=f, \quad \text { in } \Omega \\
u=\frac{\partial u}{\partial n}=0, \quad \text { on } \partial \Omega .
\end{array}\right.
$$

We choose $f$ so that the exact solution is

$$
u=\sin \left(\frac{n \pi x}{l}\right) \sin \left(\frac{\pi x}{l}\right) \sin ^{2}(\pi y) \in H_{0}^{2}(\Omega) .
$$

Our fixed-memory preconditioned GMRES algorithm [26], applied with $P_{0}$ as a preconditioner, always converged in roughly two restart cycles for $\lambda \in[0,200]$. We set the maximum dimension of the Krylov spaces to 15 .

In table 2.1 we have summarized some numerical data concerning the performance of our Fortran 77 code for the resolution of (2.9) at $\lambda=80$. As can be expected, in the solution process, the percentage of CPU time required to factorize the matrices $M$ and $P_{0}$ increases as the number of unknowns get bigger. However, since they are independent of $\lambda$, the relative cost will become lower when we shall be tracing the branches of solutions of the nonlinear problem.

\section{The nonlinear problem.}

3.1. A weak formulation. The theoretical analysis of problem (1.2) is based on its variational nature which is ensured by the following proposition (see [2]).

Proposition 3.1. The solutions of (1.2) are the critical points of the potential

$$
F(u)=\frac{1}{2}\|\Delta u\|_{0}^{2}+\frac{1}{4}\left\|\Delta\left(\Delta_{\omega}^{-2}[u, u]\right)\right\|_{0}^{2}-\frac{\lambda}{2}([\theta, u], u)_{L^{2}}-(f, u)_{L^{2}}
$$




\begin{tabular}{|l|l|l|l|l|}
\hline$\tilde{h}$ & $\frac{1}{5}$ & $\frac{1}{10}$ & $\frac{1}{15}$ & $\frac{1}{20}$ \\
\hline Number of mesh points & 641 & 2481 & 5521 & 9761 \\
\hline Number of equations & 1202 & 4802 & 10802 & 19202 \\
\hline CPU time & 11.08 & 60.24 & 193.9 & 459.18 \\
\hline Assembly and factorization: CPU time & 1.04 & 14.18 & 72.74 & 220.34 \\
\hline Assembly and factorization: \% of CPU time & 9.41 & 23.54 & 37.51 & 47.99 \\
\hline
\end{tabular}

TABLE 2.1

$l=3.9$ and $\lambda=80$ : CPU time on a SUN Ultra 1 workstation.

The Euler equation corresponding to the equilibrium point of this potential, is

$$
(\Delta u, \Delta v)_{L^{2}}-\left(\lambda[\theta, u]-\left[\Delta_{\omega}^{-2}[u, u], u\right], v\right)_{L^{2}}-(f, v)_{L^{2}}=0, \quad \forall v \in H_{0}^{2}(\Omega)
$$

which is simply the variational form of (1.2). Problem (3.1) is of the general form (2.1) and, for a given $u_{0}$, its linearization may be written

$$
(\Delta \delta u, \Delta v)_{L^{2}}-\left(\lambda[\theta, \delta u]-2\left[\Delta_{\omega}^{-2}\left[\delta u, u_{0}\right], u_{0}\right]-\left[\Delta_{\omega}^{-2}\left[u_{0}, u_{0}\right], \delta u\right], v\right)_{L^{2}}
$$

In order to follow the same route as in the linear case, we wish to express (3.1), or any linearization (3.2), in terms of the unknowns $(u, \Delta u) \in H_{0}^{1}(\Omega) \times H^{1}(\Omega)$. This will require some definitions.

- The weak deviator $\Upsilon(v)$ of a function $v \in H_{0}^{1}(\Omega)$ is defined to be the solution of the variational problem

$$
a_{2}(\Upsilon(v), \nu)+b_{2}(\nu, v)=0, \quad \forall \nu \in \Sigma_{D}(\mathcal{X})
$$

- Given two pairs

$$
X_{1}=\left(u_{1}, w_{1}\right) \text { and } X_{2}=\left(u_{2}, w_{2}\right) \in H_{0}^{1}(\Omega) \times H^{1}(\Omega),
$$

and $\mu_{i}=\Upsilon\left(u_{i}\right), i=1,2$ the corresponding solutions of (3.3), we define the weak Airy triplet

$Y\left(X_{1}, X_{2}\right)=\left(\phi\left(X_{1}, X_{2}\right), \omega\left(X_{1}, X_{2}\right), \mathcal{M}\left(X_{1}, X_{2}\right)\right) \in H_{0}^{1}(\Omega) \times H^{1}(\Omega) \times \Sigma_{D}\left(H^{1}(\Omega)\right)$

in the following way. The pair $\left(\phi\left(X_{1}, X_{2}\right), \omega\left(X_{1}, X_{2}\right)\right)$ is the solution of the problem

(3.4) $\left\{\begin{array}{l}a_{1}\left(\omega\left(X_{1}, X_{2}\right), \psi\right)+b_{1}\left(\psi, \phi\left(X_{1}, X_{2}\right)\right)=0, \forall \psi \in H^{1}(\Omega) \\ b_{1}\left(\omega\left(X_{1}, X_{2}\right), v\right)=\int_{\Omega} \llbracket \frac{1}{2} w_{1} I_{2}+\mu_{1}, \frac{1}{2} w_{2} I_{2}+\mu_{2} \rrbracket v d \underline{x}, \forall v \in H_{0}^{1}(\Omega) .\end{array}\right.$

whereas $\mathcal{M}\left(X_{1}, X_{2}\right)$ is that of $(3.3)$ when $v=\phi\left(X_{1}, X_{2}\right)$.

With these definitions, we can model the nonlinear coupling term, $\left(\left[\Delta_{\omega}^{-2}[u, u], u\right], v\right)_{L^{2}}$ and our weak version of (3.1) is the Miyoshi-like nonlinear problem: find

$$
\mathbf{X}=(u, w) \in H_{0}^{1}(\Omega) \times H^{1}(\Omega)
$$

such that

$$
(3.5)\left\{\begin{array}{l}
a_{1}(w, \psi)+b_{1}(\psi, u)=0, \forall \psi \in H^{1}(\Omega) \\
b_{1}(w, v)-\int_{\Omega}\left(\lambda \llbracket H(\theta), \frac{1}{2} w I_{2}+\Upsilon(u) \rrbracket\right) v d \underline{x} \\
\left.+\int_{\Omega} \llbracket \frac{1}{2} \omega(X, X) I_{2}+\mathcal{M}(X, X), \frac{1}{2} w I_{2}+\Upsilon(u)\right]
\end{array}\right.
$$$$
\text { where } H(\theta) \text { is the Hessian of } \theta \text {. }
$$ 
3.2. Discretization. The discretization of (3.5) is based on the same finite element spaces as that of (2.5). The displacement $u$ and Airy Stress tensor $\phi=\Delta^{-2}[u, u]$ will be discretized by function in $\mathcal{V}_{h}$; the Laplacians $w$ and $\omega$ by functions in $\mathcal{X}_{h}$ and the deviators $\Upsilon$ and $\mathcal{M}$ by functions of $\Sigma_{D}\left(\mathcal{X}_{h}\right)$. The corresponding discretized versions of (3.3), (3.4), (3.5) are then obtained in a straightforward way.

3.3. The algorithm. Using the nodal values vector $\mathbf{X}_{h}=\left(U_{h}, W_{h}\right) \in \mathbb{R}^{N_{\mathcal{V}_{h}} \times}$ $\mathbb{R}^{N_{\mathcal{X}_{h}}}$ of the discrete solution $\left(u_{h}, w_{h}\right)$, problem (3.5) can be written as an algebraic system of equations:

Find $\mathbf{X}_{h}=\left(U_{h}, W_{h}\right) \in \mathbb{R}^{N_{\mathcal{V}_{h}}} \times \mathbb{R}^{N_{\mathcal{X}}}$ such

$$
\mathcal{F}\left(\mathbf{X}_{h}, \lambda\right)=0
$$

There are various ways of constructing a Newton-GMRES algorithm to solve the above problem. Our approach is based on three remarks.

1. We have defined the problem in order to reduce the number of primary unknowns and thus the size of the tangent matrix.

2. If one looks at the linearized version of the problem: $X_{n}=\left(u_{n}, w_{n}\right)$ being given, find $\delta X_{n}=\left(\delta u_{n}, \delta w_{n}\right)$ such that

$$
\left\{\begin{array}{l}
a_{1}\left(\delta w_{n}, \psi\right)+b_{1}\left(\psi, \delta u_{n}\right)=a_{1}\left(w_{n}, \psi\right)-b_{1}\left(\psi, u_{n}\right), \quad \forall \psi \in \mathcal{X}_{h} \\
b_{1}\left(\delta w_{n}, v\right)-\lambda \int_{\Omega} \llbracket H(\theta), \frac{1}{2} \delta w_{n} I_{2}+\Upsilon\left(\delta u_{n}\right) \rrbracket v d \underline{x} \\
\quad+\int_{\Omega} \llbracket \frac{1}{2} \omega\left(X_{n}, X_{n}\right)+\mathcal{M}\left(X_{n}, X_{n}\right), \frac{1}{2} \delta w_{n} I_{2}+\Upsilon\left(\delta u_{n}\right) \rrbracket d \underline{x} \\
\quad+2 \int_{\Omega} \llbracket \frac{1}{2} \omega\left(\delta X_{n}, X_{n}\right) I_{2}+\mathcal{M}\left(\delta X_{n}, X_{n}\right), \frac{1}{2} w_{n} I_{2}+\Upsilon\left(u_{n}\right) \rrbracket v d \underline{x} \\
=b_{1}\left(w_{n}, v\right)+\lambda \int_{\Omega} \llbracket H(\theta), \frac{1}{2} w_{n}+\Upsilon_{n} \rrbracket v d \underline{x}+\int_{\Omega} \llbracket \frac{1}{2} \omega\left(X_{n}, X_{n}\right) I_{2} \\
\quad+\mathcal{M}\left(X_{n}, X_{n}\right), \frac{1}{2} w_{n} I_{2}+\Upsilon_{n} \rrbracket v d \underline{x}+\int_{\Omega} f v d \underline{x}, \quad \forall v \in \mathcal{V}_{h}
\end{array}\right.
$$

one sees that the computation of residues is not much more complicated for that version than for the problem itself.

3. As seen in section 2.4, when applied to (2.8), the convergence of GMRES is very fast.

Thus, neither the size of the problem nor its complexity requires that we avoid explicit differentiation. For all these reasons, we have decided not to use nonlinear variants of GMRES but rather to embed it into the Newton iterations and to let it converge at each step. The resulting algorithm can be described in the following basic form

Let $X_{0}=\left(u_{0}, w_{0}\right)$ be given, for $n=0,1, \ldots$ until convergence do

1. Compute $\Upsilon_{n}$ by solving (3.3).

2. Compute $Y\left(X_{n}, X_{n}\right)$ in two steps

(a) Compute $\left(\phi\left(X_{n}, X_{n}\right), \omega\left(X_{n}, X_{n}\right)\right)$ by solving (3.4)

(b) Compute $\mathcal{M}\left(X_{n}, X_{n}\right)$ by solving (3.3) with $v=\phi\left(X_{n}, X_{n}\right)$.

3. Compute $\left(\delta u_{n}, \delta w_{n}\right)$ by using GMRES to solve (3.6) with a preconditioning matrix equal to $P_{0}$. For each search direction, one must compute one new 
Airy triplet $Y\left(X_{n}, \delta X_{n}\right)$ and one new weak deviator $\Upsilon\left(\delta u_{n}\right)$. This is done by repeating step 1 and 2 above.

4. Set $\left(u_{n+1}, w_{n+1}\right)=\left(u_{n}, w_{n}\right)+\left(\delta u_{n}, \delta w_{n}\right)$.

Traditional strategies for controlling the length of the Newton step for robustness do not seem to be required.

With this choice, our algorithm will be based on the use of only two matrices, namely the mass matrix $M$ of the $L^{2}(\Omega)$ inner product restricted to $\mathcal{X}_{h}$, and the "biharmonic matrix" $P_{0}$ of problem $(2.9)$ when $\lambda=0$. The first is used to compute the components of deviators whereas the second is used to compute Airy triplets and as a preconditioner. These matrices are factorized at the beginning of the continuation process, so that each intermediate linear resolution is done at a cost which is of the same order as that of a matrix-vector product.

REMARK 3.2.

1. In the coming sections, the above algorithm is embedded into the classical Euler-Newton continuation procedure. The extra terms appearing in the residual of the augmented system are all linear in $(u, w)$ and their computation requires nothing more than scalar products. As to the preconditioner, it is simply augmented with the last line of the identity matrix of appropriate size.

2. Our algorithm is, in a sense, of the same family as the one proposed by Huitfeldt and Ruhe in [16]. However, here we have reduced the number of primary unknowns and we use the same preconditioner along every branch.

3. In discussing memory requirements, one is mainly interested in the size of the matrices which, in turn is dictated by the number of unknowns. Here we have two scalar unknowns, the displacement $u$ and the trace of the Hessian $\Delta u$. This is comparable to the choice made in [24] and [8] where the variables are the displacement $u$ and the Airy potential $\phi$. Of course memory is also a function of the matrix storage. Here we use a skyline storage since CSR is not compatible with $L U$-factorization.

4. Typically for the numerical results presented in section 5 below, we set the dimension of the Krylov spaces to 20 on the branches of symmetric solutions and 30 on the branches of non-symmetric solutions.

5. As explained before, the only work with which convergence comparison can really be made is [24]. Since the choice of the discretization has a direct impact of the conditioning of the matrices, we can only comment on the respective robustness of the two approaches. One can find in [24] a bifurcation diagram on which the number of iteration is given in terms of $\lambda$. It is quite clear on this diagram that the conjugate gradient is very sensitive to singular point since the number of iterations will often double near them. In our case, Newton method applied to the augmented system is insensitive to singularity but the linear system is somewhat stiffer.

4. On the detection of bifurcation points. In order to obtain a complete description of the bifurcation diagram, one needs some numerical means of detecting singular points along solution curves. In the classical Euler-Newton approach, this is simply done by monitoring the determinant of the augmented tangent matrix (see $[1])$.

If one uses an iterative approach this is not operational since that tangent matrix is never constructed or factorized. In [16] the authors propose to rely on the spectrum of the Hessenberg matrix produced by the Arnoldi process to monitor bifurcations. In the same vein Chien et al. [8] suggest to monitor the condition number of the reduced 
matrix. We have tested the first idea but found that it sometimes unexpectedly fails by producing spurious negative eigenvalues for reasons not easily discernable. Instead of pursuing that direction we prefer to follow an idea of Damil, Potier-Ferryet al. (see [28], [4], or Cadou [5]), who suggest that one can detect bifurcation by monitoring a scalar quantity, called a detector, which changes sign at the same points on the curves as the determinant. This is a particular case of a more general approach for the computation of quadratic bifurcation point with the help of Moore-Spence system. A general description is given in [15, section 4.1], thus we shall only briefly describe the idea and refer the reader to the above quoted works for further practical details.

Let there be given a nonlinear problem $F(u, \lambda)=0$ and a solution branch that starts at a point $\left(u_{0}, \lambda_{0}\right)$. Suppose that the two vectors $h$ and $g$ are such that $h^{t} D_{u} F\left(u_{0}, \lambda_{0}\right)^{-1} g \neq 0$ and that $\alpha_{0}$ is a non-zero scalar. Then, if for each $\lambda$, $(v(\lambda), \mu(\lambda))$ denote the solution of

$$
\left[\begin{array}{cc}
D_{u} F(u, \lambda) & g \\
h^{t} & 0
\end{array}\right]\left[\begin{array}{l}
v(\lambda) \\
\mu(\lambda)
\end{array}\right]=\left[\begin{array}{c}
0 \\
\alpha_{0}
\end{array}\right],
$$

the sign changes of $\operatorname{det}\left(D_{u} F(u, \lambda)\right)$ coincide with those of $\mu(\lambda)$ as long as $h^{t} D_{u} F(u, \lambda)^{-1} g$ remains non-zero along the branch. Thus, the computation of the detector $\mu$ requires the resolution of a linear system of the same type as the augmented tangent system of our nonlinear problem, the only difference being in the bordering vectors.

It was a simple procedure to modify our iterative algorithm to solve system (4.1). It proved to be a rather inexpensive and reliable approach. However, when considered as a function of $\lambda$, the detector can have poles when $h^{t} D_{u} F(u, \lambda)^{-1} g=0$ and thus change sign at a point where the determinant does not. We have observed that, even for randomly chosen $g$ and $h$, this is not infrequent and that this has to be carefully analyzed. The result of this analysis and some proposal of remedy will be presented in a forthcoming paper.

5. Numerical results. We come to the presentation of the numerical results. We shall limit our attention to two specific applications. In the first case, we shall review some results on multiple bifurcations obtained by Chien and Chen [7] while in the second we shall analyze the mode jumping phenomenon in the case of a fully clamped plate compressed on its four sides, a case which was not covered in [13]. In each case, we shall compute bifurcation diagrams and solutions on the various branches. But first let us start with some preliminaries.

5.1. Preliminaries. In this section we set $f=0$. As is well known, when $\lambda$ is small, the only solution of system (1.1) is the solution $u=0$. The subset $(\lambda, 0)$ of the bifurcation diagram will be called the trivial branch. Starting anywhere on that branch and varying $\lambda$, one will encounter values $\lambda_{0}$ past which the plate buckles to a configuration with a certain number $k$ of maxima and minima which depends $\lambda_{0}$ and on its length. The corresponding branches of the bifurcation diagram will be called primary branches whereas those which might bifurcate from them will be called secondary. Mathematically, a buckling load $\lambda_{0}$ corresponds to an eigenvalue of the linearized operator, which, in the case of the trivial solution, is the biharmonic operator. When the plate is simply supported $(u=\Delta u=0$ on $\Gamma)$, the eigenvalues and eigenvectors are given by 


$$
\begin{aligned}
\lambda_{p, q} & =\left(\frac{\ell \pi}{p}\left(\left(\frac{p}{\ell}\right)^{2}+q^{2}\right)\right)^{2}, \\
U_{p, q} & =\sin \left(\frac{p \pi x}{\ell}\right) \sin (q \pi y) .
\end{aligned}
$$

No such general formula exists for other combinations of boundary conditions, but the geometric aspect of the solutions will always be the same and we shall use the same notation. A simple analysis based on (5.1) shows that, for some specific lengths, which we shall call critical, the first eigenvalue is double. This is true for other boundary conditions. In that case, the post-buckling behavior can be quite complicated, the most interesting situation corresponding to the so called "mode jumping" phenomenon extensively studied by Schaeffer and Golubitsky [27]. Briefly stated, in that case, for a length $\ell$ near the critical one there will be two primary branches of solutions and a small secondary one which connects them near the bifurcation point.

Several numerical methods have been developed and used to trace the solution branches of the von Kármán equations (see Reinhart [24], [23], Miyoshi [19], Oukit [21]). However few such efforts have been carried out to investigate the mode interaction (see Gervais, Oukit and Pierre [13], Chien and Chen [7]). Here we shall limit ourselves to the direct approach which consists in the tracing of the diagram itself since it reveals traits uncovered by the local analysis of Schaeffer and Golubitsky.

To this end, all the bifurcation diagrams computations were conducted with a Fortran 77 code in which we implemented the classical Euler-Newton continuation procedure in which all systems were solved with our iterative algorithm and the bifurcation points detected as explained in section 4 . This code was run on a SUN Ultra 1 workstation.

In view of the dominant role of symmetry, all the computations were conducted on meshes for which the discrete solutions exhibit the same symmetries as those of the solution of the continuous problem. These meshes were easily constructed by first partitioning the computation domain in equal sub-squares which were then split in four equal triangles using the diagonals.

5.2. Simple secondary bifurcations for von Kármán problem. In this subsection, we shall briefly review some computations presented in [7] concerning secondary bifurcations in the neighborhood of a double bifurcation point in the case of simply supported boundary conditions. More precisely, Chien and Chen considered the problem

$$
\left\{\begin{array}{l}
\left.\Delta^{2} u=[\phi, u]-\lambda \frac{\partial^{2} u}{\partial x^{2}}, \quad \text { in } \Omega=\right] 0,2[\times] 0,1[, \\
\left.\Delta^{2} \phi=-\frac{1}{2}[u, u], \quad \text { in } \Omega=\right] 0,2[\times] 0,1[, \\
u=\Delta u=0, \quad \text { on } \partial \Omega, \\
\phi=\Delta \phi=0, \quad \text { on } \partial \Omega .
\end{array}\right.
$$

Consideration of (5.1) with $\ell=2$ shows that the third bifurcation on the trivial branch is double and corresponds to $\lambda_{1,1}=\lambda_{4,1}=61.6850275068$ and $U_{1,1}=\sin (\pi x / 2) \sin (\pi y) U_{4,1}=\sin (2 \pi x) \sin (\pi y)$.

In order to get a more complete view of the bifurcation diagram than that proposed in [7], and in particular of the so called symmetry breaking solutions, we have 
used our code on a mesh of 800 elements for which the number of degrees of freedom in $(u, \Delta u)$ is 3082. Of course, since the discrete problem is a perturbation of the continuous one and since perturbation generically splits multiple eigenvalues, setting $\ell=2$, we did not get a double eigenvalue but rather two very close simple ones $\lambda_{1}=61.685084$ and $\lambda_{2}=61.691933$ (see figure 5.1). In our computations, the symmetry breaking solutions do not bifurcate from the trivial branch but rather appear as a secondary bifurcation on $U_{4,1}$ branch at $\lambda=61.6942301$. Chien and Chen indicated also that they have detected a bifurcating point on that branch and none on $U_{1,1}$. In figures 5.2 we present a view of $U_{1,1}$ and $U_{4,1}$ while in figure 5.3 we exhibit the variation of the symmetry breaking solutions when $\lambda$ first decreases down to the bifurcation value 61.6942301 and then starts to increase again. Here the non-symmetry is much clearer than that illustrated by [7].

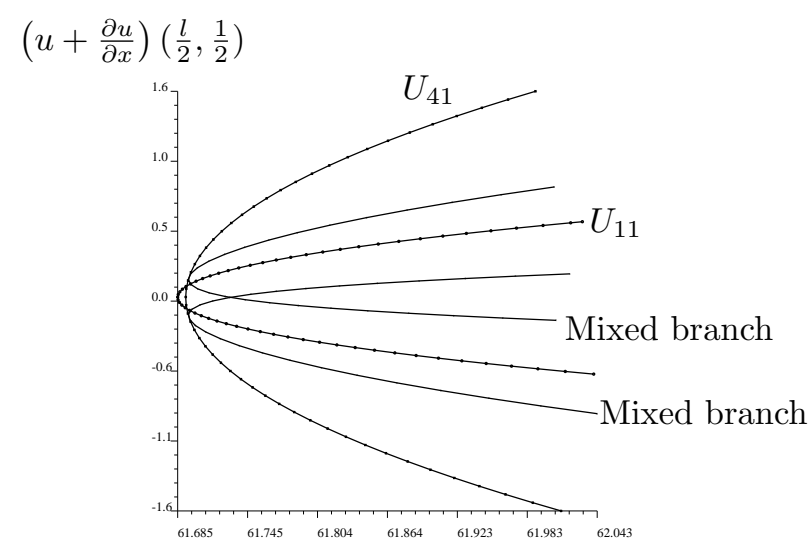

FIG. 5.1. Simply supported plate: bifurcation diagram $(l=2)$.
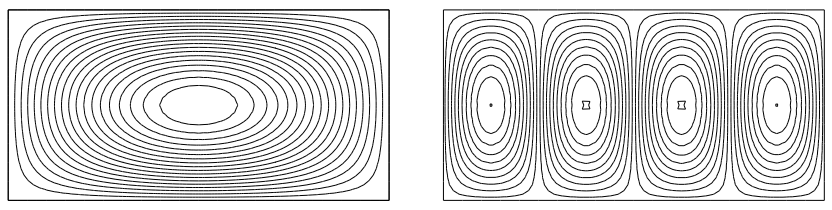

FIG. 5.2. Simply supported plate: $U_{1,1}$ and $U_{4,1}$.

5.3. Mode interaction for a fully clamped plate compressed on its four sides. In this last section, we propose a complete analysis of the bifurcation diagram of problem (1.1), in the neighborhood of a double bifurcation point. This analysis reveals strong differences with respect to the cases studied in [27] or [13] of a plate either partly clamped or fully clamped but compressed only on two sides. According to the theory exposed in [27] these differences are to be attributed to the modifications of the boundary conditions both for $u$ and $\phi$ and to the fact that the normal compressive force is exerted along the whole boundary. We remark that this case was also the test case chosen by Reinhart but that she used coarse meshes and limited herself to the analysis of the first bifurcation without paying attention to the post-buckling behavior.

First we remark that, in the case under consideration, the eigenfunctions have four tiny nodal domains located on each corner of the rectangle $\Omega$. This was already 


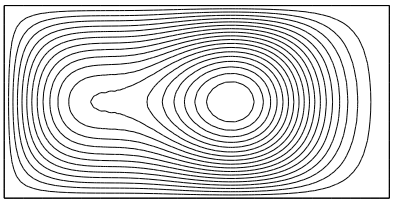

$\lambda=61.7294$

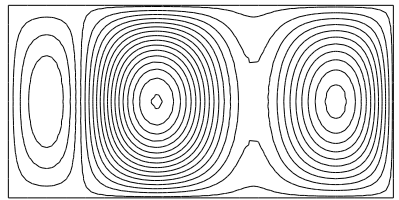

$\lambda=61.6953$

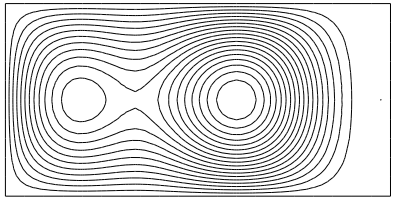

$\lambda=61.7042$

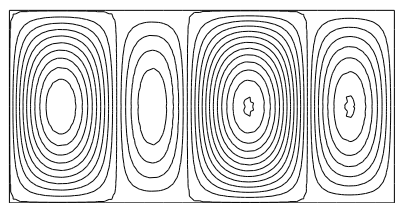

$\lambda=61.6943$

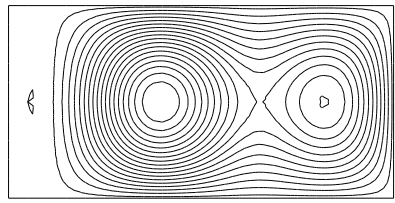

$\lambda=61.7002$

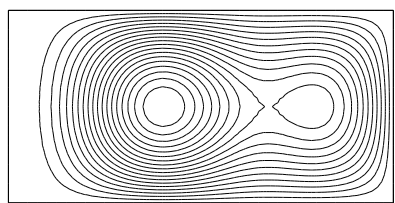

$\lambda=61.7089$

FIG. 5.3. Simply supported plate: Transition from non-symmetric mode to symmetric mode $U_{4,1}$.

observed in [18], where Kozlov, Kondrat'ev and Maz'ya studied this corner effect for the eigenvectors of the biharmonic problem $\Delta^{2}=\lambda \Delta u$, with built-in boundary condition $u=\frac{\partial u}{\partial n}=0$, on rectangular domain. However, these corner nodal domains are usually small compared to the others (see figure 5.4) and it still makes sense to use the $U_{p, q}$ notation.

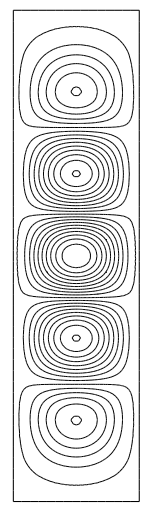

Level lines

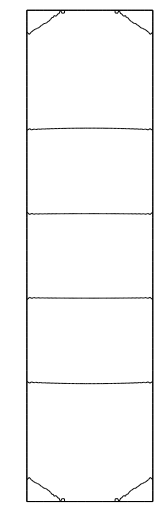

Nodal lines

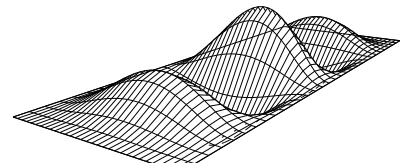

three-dimensional view

FIG. 5.4. Corner effect: the values of the solution on the four nodal domains located on the edge of the rectangle are so small that they do not even appear on the level lines or 3-dimensional view.

As pointed out before, there is no known analytical formula for the eigenvalues of the fully clamped plate but, from the numerical point of view, their determination is not a problem. In [13] we showed that the same FE discretization can be used to solve the appropriate spectral problem $\Delta^{2} u=-\lambda \Delta u$. Thus, using that code, we carefully computed the first eigenvalues for different length $\ell$ and found a sequence of critical lengths $\ell^{*}$ for which the first two eigenvalues coincide (see figure 5.5).

For instance there exists a critical length $\ell^{*}$ satisfying $7.9<l^{*}<7.97$ and for which the dominating modes are $U_{5,1}$ and $U_{6,1}$. This case will serve as an illustration of our findings. Here the computations were conducted on a mesh of 1008 elements which corresponds to $4034(u, \Delta u)$ degrees of freedom. 


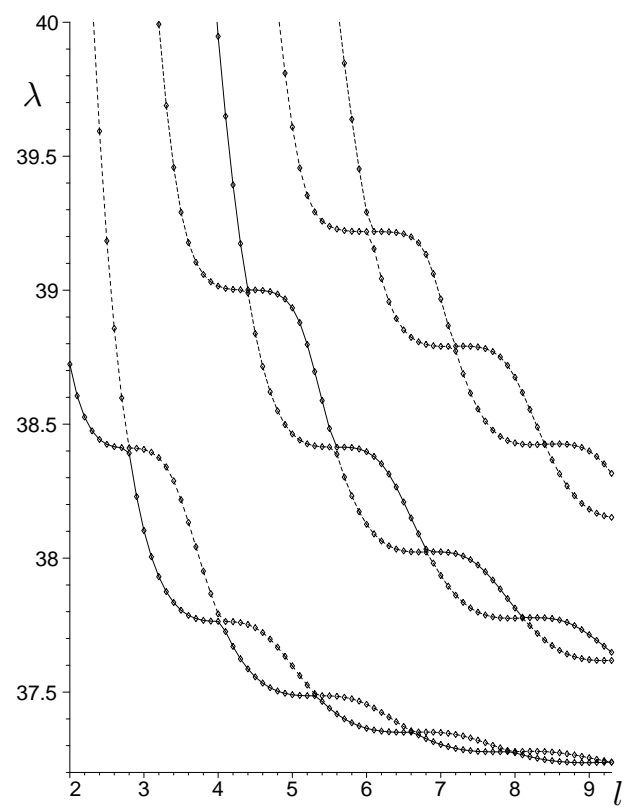

FIG. 5.5. Curves of the first six bifurcation values vs. $\ell$ : for $4.1 \leq \ell \leq 5.3$ the dominant mode is $U_{3,1}$, for $5.4 \leq \ell \leq 6.6$ the dominant mode is $U_{4,1}$, for $6.7 \leq \ell \leq 7.9$ the dominant mode is $U_{5,1}$, for $8 \leq \ell \leq 9.2$ the dominant mode is $U_{6,1}$.

Let us start with the case $l=7.9<\ell^{*}$ which is described by figures 5.6, 5.7 and 5.8. We remark that

- The first branch corresponds to $U_{5,1}$ (see figure 5.7).

- Following the second primary branch $\left(U_{6,1}\right)$, we pass two successive limit points through which the solutions loose two nodal domains (see figure 5.8).

- These points being passed, we meet a secondary bifurcation on a closed loop of non-symmetrical solutions, connecting the present mode of type $U_{4,1}$ to a mode of type $U_{5,1}$.

- If we rather follow the $U_{6,1}$ branch, we meet the above secondary one and then, soon afterward, a sequence of limit points. There is not any mode interaction between the dominating mode $U_{5,1}$ and the second one $U_{6,1}$

The case $l=7.97>\ell^{*}$ is described by figures 5.9 and 5.10 .

- Following any of the two branches, we first meet a secondary loop of nonsymmetrical solutions connecting the principal branches. Thus there is mode interaction (mode jumping) between the dominating $U_{6,1}$ mode and the second $U_{5,1}$ mode (see figures 5.9 and 5.10).

- Past this first loop, the diagram is equivalent to the one described for $\ell<\ell^{*}$. In particular, the two close limit points, through which nodal domains are lost, are still present.

In figure 5.11 we show some solutions on the branch of non-symmetric solutions (mixed branch) near the point $\mathrm{P}$ of figure 5.6 where this branch intersects the branch of $U_{4,1}$ solutions. We can clearly see that the small nodal domains in the corners play an important role in the mode interaction process. 


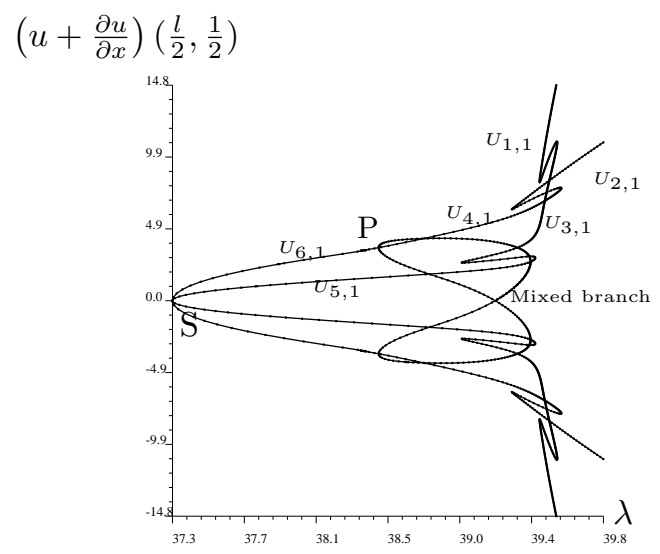

FIG. 5.6. $l=7.9$ : Bifurcation diagram.

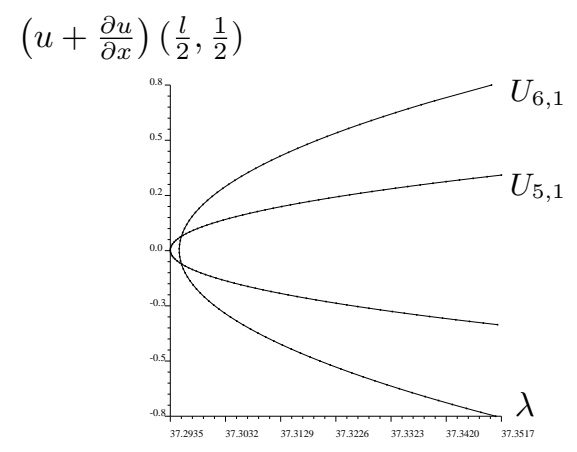

FIG. 5.7. Zoom around point $S$ on figure 5.6: It is the $U_{5,1}$ mode which corresponds to the first bifurcation value.

These observations would be valid for other critical lengths $\ell^{*}$ (see [11]). Thus the situation is in contrast with the one described in [13] for a clamped plate compressed on its two ends.

Let us summarize the main differences.

- If $\ell^{*}$ is a critical length then mode jumping occurs only for $l>\ell^{*}$, in contrast to the case of the plate with simply supported boundary condition on its two edges and built-in and compressed on its two ends (see [27]), or of the plate with built-in boundary condition on its four sides and pushed on its two ends (see [13]), where mode jumping rather occurs for $l<\ell^{*}$.

- Along primary branches the number of nodal domains decreases. For instance if the first primary branch has $U_{n, 1}$ type solutions $(n \geq 3)$ when it bifurcates from the trivial branch, then along that branch the solutions lose two nodal domains (without breaking of symmetry) to become $U_{n-2,1}$ and this change occurs by transitioning through two limit points. If $n-2 \geq 3$, the same process will repeat.

- After those two limit points there will always be a secondary loop connecting the $U_{n-2,1}$ branch to the $U_{n-1,1}$ one.

Here one important question remains to be settled: are these distinct bifurcation 


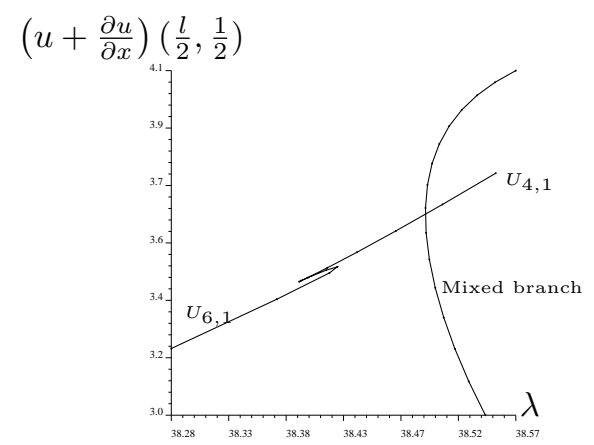

FIG. 5.8. Zoom near point $P$ on figure 5.6: There are two limit points through which the solutions change from $U_{6,1}$ to $U_{4,1}$

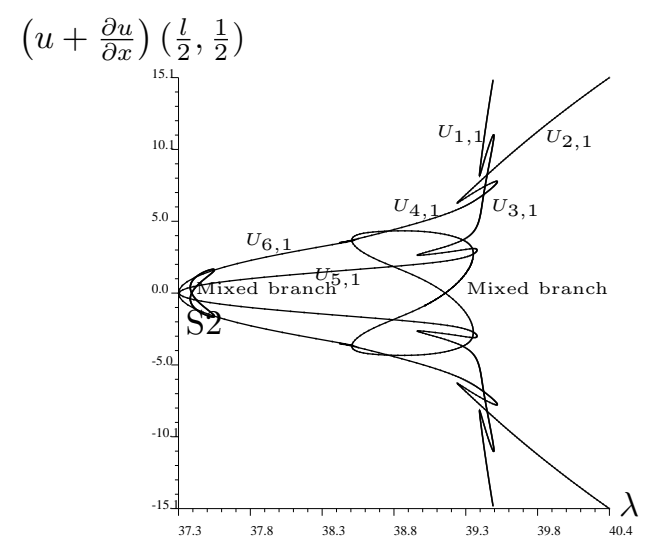

FIG. 5.9. $l=7.97$ : Bifurcation diagram

features related to the continuous problem or do they occur because of some imperfection of the discretization? To obtain an experimental answer, we have repeated the computation of the diagram presented below on finer finite element meshes and observed that all the noted features were persistent [11]. This suggests that the convergence of the discrete diagram to the continuous one preserves topology.

6. Conclusion. In this paper we have proposed a new continuation algorithm for the resolution of the von Kármán system. This algorithm consists in modifying the correction step of the classical Euler-Newton continuation procedure in which one replaces the resolution of two linear systems which follows from the application of a block LU factorization, by an iterative resolution of the bordered linearized system. For this we have selected a preconditioned GMRES algorithm in which the preconditioner is fixed along any branch of solutions. This preconditioner is simply the discretization of the the tangent operator corresponding to the trivial solution and leads to a well conditioner iteration matrix.

After a validation of the algorithm and a few comparisons with others of a similar nature, we have proposed a new analysis of the post-buckling behavior of a clamped rectangular plate compressed along its whole boundary. This analysis reveals interesting differences with those presented elsewhere, thus confirming that the post-buckling 


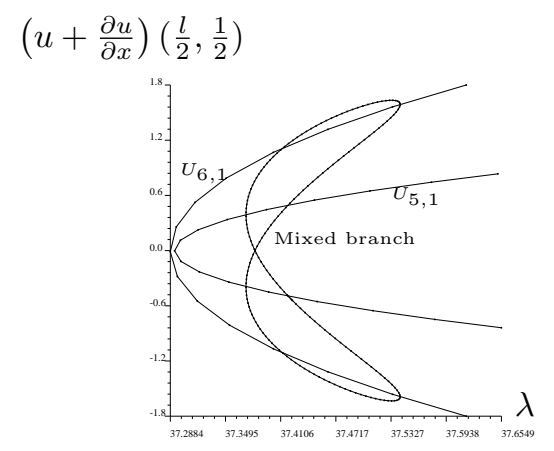

FIG. 5.10. $l=7.97:$ Zoom near point S2

behavior is strongly related to the boundary conditions and the form of the compression.

Acknowledgement. The authors would like to thank one of the referees for many useful remarks that greatly helped to improve the manuscript. 


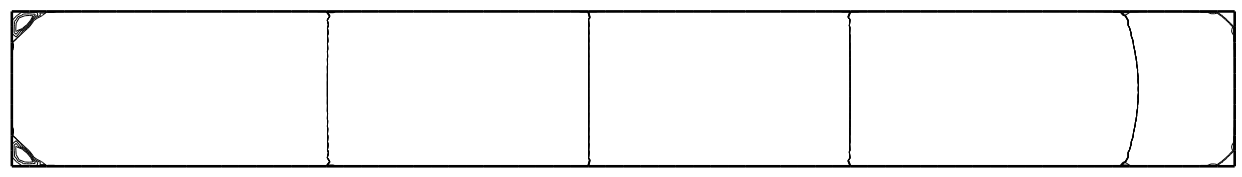

$\lambda=38.615$

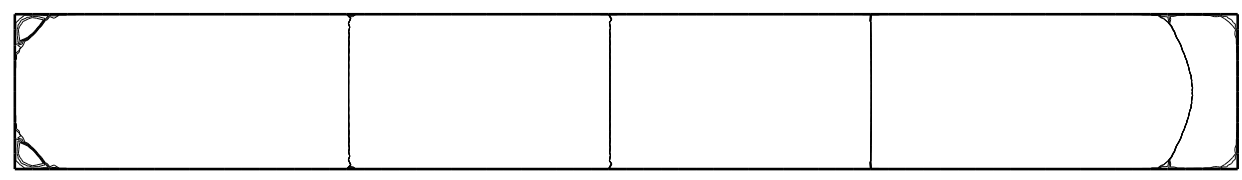

$\lambda=38.507$

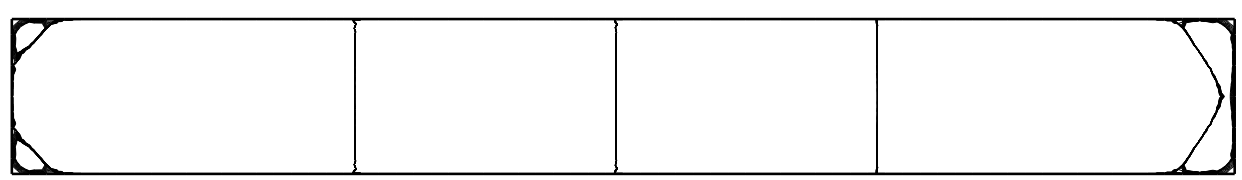

$\lambda=38.484$

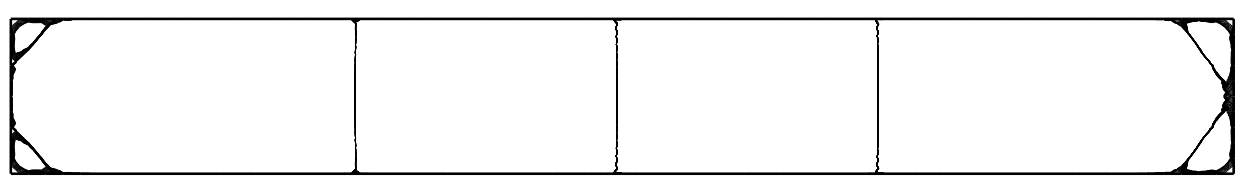

$\lambda=38.482$

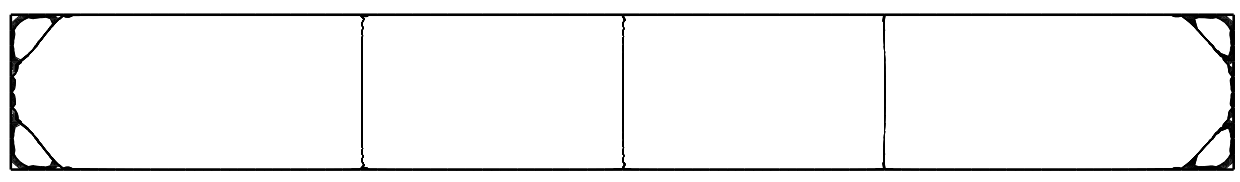

$\lambda=38.479$

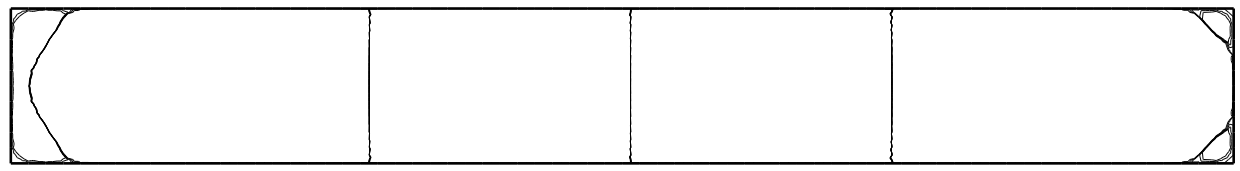

$\lambda=38.483$

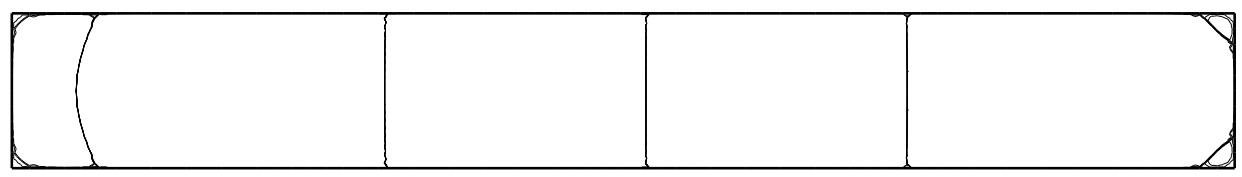

$\lambda=38.534$

FIG. 5.11. Transition from non-symmetric mode to symmetric mode $(\lambda=38.479)$. 


\section{REFERENCES}

[1] E. L. Allgower And K. Georg, Numerical continuation methods, Springer-Verlag, Berlin, 1990.

[2] M. S. Berger, On von Kármán's equations and the buckling of a thin elastic plate. I. The clamped plate, Comm. Pure Appl. Math., 20 (1967), pp. 687-719.

[3] I. Bock On large deflections of viscoelastic plates. Math. Comput. Simulations, 50 (1999), no. 1-4: $135-143$.

[4] H. B. Boutyour, B. Cochelin, And M. Potier-Ferry, Calculs des points de bifurcation par une méthode asymptotique numérique, in $1^{\mathrm{er}}$ Congrès National de Mécanique au Maroc, 1993, pp. 371-378.

[5] J.-M. CADOU, Méthode asymptotique numérique pour le calcul des branches solutions et des instabilités dans les fluides et pour les problèmes d'interaction fluide-structure, $\mathrm{PhD}$ thesis, Université de Metz, Metz, France, 1997.

[6] T. F. CHAN AND Y. SAAD, Iterative methods for solving bordered systems with applications to continuation methods. SIAM J. Sci. Statist. Comput. 6 (1985), no. 2, 438-451.

[7] C. S. Chien And M.-S. Chen, Multiple bifurcation in the von Kármán equations, SIAM J. Sci. Comput., 18 (1997), pp. 1737-1766.

[8] C. S. Chien, S. L. Chang And Z. MeI, Tracing the buckling of a rectangular plate with a Block GMRES method J. Comput. Appl. Math. 136 (2001), no. 1-2, 199-218.

[9] C. S. Chien, S. Y. Gong and Z. MeI, Mode jumping in the von Kármán equations SIAM J. Sci. Comput., 22 (2000), no. 4, pp. 1354-1385

[10] P. G. Ciarlet and P. Rabier, Les équations de von Kármán, Springer, Berlin, 1980.

[11] K. Dossou, Résolution numérique des équations de von Kármán, $\mathrm{PhD}$ thesis, Université Laval, Québec, QC Canada, 2000.

[12] R. S. FAlK AND J. E. Osborn, Error estimates for mixed methods, R.A.I.R.O. Anal. Numer, 14 (1980), pp. 249-277.

[13] J.-J. Gervais, A. Oukit, And R. Pierre, Finite element analysis of the buckling and mode jumping of a rectangular plate, Dynam. Stability Systems, 12 (1997), pp. 161-185.

[14] M. Golubitsky and D. G. Schaeffer, Singularities and groups in bifurcation theory. Vol. I, vol. 51 of Applied Mathematical Sciences, Springer-Verlag, New York, 1985.

[15] W. J. F. GovaerTs, Numerical Methods for Bifurcations of Dynamical Equilibria, SIAM, 2000.

[16] J. Huitfeldt and A. Ruhe, A new algorithm for numerical path following applied to an example from hydrodynamical flow, SIAM J. Sci. Stat. Comput., 11 (1990), pp. 1181-1192.

[17] H. KELLER, Numerical solution of bifurcation and nonlinear eigenvalue problem, in Applications of bifurcation theory, P. H. Rabinowitz, ed., Academic Press, New-York, 1977.

[18] V. A. Kozlov, V. A. KondraT'Ev, And V. G. MAZ'YA, On sign variation and the absence of "strong" zeros of solutions of elliptic equations, Math. USSR-Izv., 34 (1990), pp. 337-353.

[19] T. Miyoshi, A mixed finite element method for the solution of the von Kármán equations, Numer. Math., 26 (1976), pp. 255-269.

[20] M. Ortiz AND G. Gioia, The morphology and folding patterns of buckling-driven thin-film blisters, J. Mech. Phys. Solids, 42, no. 3:531-559, 1994.

[21] A. Oukit, Étude théorique et résolution numérique des équations de Von Kármán, $\mathrm{PhD}$ thesis, Université Laval, Québec, QC Canada, 1996.

[22] A. Oukit And R. Pierre, Mixed finite element for the linear plate problem: the HermannMiyoshi model revisited, Numer. Math., 74 (1996), pp. 453-477.

[23] L. Reinhart, Sur la résolution numérique de problèmes aux limites non linéaires par des méthodes de continuation, PhD thesis, Université Pierre et Marie Curie, Paris VI, 1980. Thèse de 3ème cycle.

[24] - On the numerical analysis of the von Kármán equations: mixed finite element approximation and continuation techniques, Numer. Math., 39 (1982), pp. 371-404.

[25] E. Riks, C. Rankin, F.A. Brogan, On the solution of mode jumping phenomena in thin walled structures, Comput. Meth. Appl. Mech. Engrg. 136 (1996), pp. 59-92.

[26] Y. SAAD AND M. H. SCHULTZ, GMRES: a generalized minimal residual algorithm for solving nonsymmetric linear systems. SIAM J. Sci. Statist. Comput. 7 (1986), no. 3, 856-869.

[27] D. Schaeffer and M. Golubitsky, Boundary conditions and mode jumping in the buckling of a rectangular plate, Comm. Math. Phys., 69 (1979), pp. 209-236.

[28] P. Vannucci, B. Cochelin, N. Damil, and M. Potier-Ferry, An asymptotic-numerical method to compute bifurcating branches, Internat. J. Numer. Methods Engrg., 41 (1998), pp. $1365-1389$.

[29] J. Weimin and P. Sternberg, Energy estimates for the von Kármaán model of thin-film blistering J. Math. Phys., 42, no.: 192-199, 2001. 